have been overlooked if the attention of the radiologist had not been focused for some time on this disease. In the absence of the skull radiopacities, the age of the patient might have led one to omit the $x$-ray examination of the limbs. It would seem that the investigation for the cause of epilepsy or allied mental and psychical conditions cannot be regarded as complete until a thorough search has been made in the soft tissues for the presence of the calcified cysticercus. It is imperative for the radiologist to be acquainted with the possible appearances of the calcified or early calcifying cysticercus. The figure [reduced to 2/5 linear] shows the various shapes seen in the radiographs of twelve cases in this hospital. Their actual size can be calculated from the millimetre scale reproduced at the right - hand lower corner.

\section{Calcification of the} Cysticercus

Calcification of the cysticercus in the brain is rare in our series of cases, and was found in only one other out of twelve. At present radiology can play a part in the diagnosis only when calcium is deposited in the dead parasite-that is, relatively late in the disease. It is to be noted that the presence of calcified cysticerci

by no means excludes the presence of living parasites in the same patient. The amount of calcium deposit and the time taken for this depend on various factors not yet fully understood. The radiopacities in the tissues in a case of cysticercosis represent: the calcifying or calcified scolex, the true bladder in which it developed, the remains of the fluid contents of the true bladder, the outer cyst wall provided by the tissues of the host, and the remains of the fluid contents of this outer cyst. The shape depends on the pressure by neighbouring structures.

The scolex, as the solid portion of the bladder-worm, may be expected to receive the heaviest deposit, and this fact is well illustrated in Shape 4 (" oval with spot"), which in the radiograph exactly resembles the excised cysticercus with its glistening " milk-spot" on the bladder wall. Caseation before calcification will explain the appearance of the lines indicated in the various diagrams. Many of the calcified cysticerci show a very definite halo, which is accepted as indicating calcification in the outer cyst wall or in its outside layers.

The skull radiograph is often asked for in a case of epilepsy, but if there is the least reason to suspect cysticercosis -it would be much better to devote the radiographic examination to the soft tissues. The routine in such a case at present is: lateral view of the skull, root of neck, upper arms, forearms, thighs, and legs.

\section{Diagnosis}

The figure showing the various shapes indicates what the radiologist has to search for in the tissues. The names are given for easy reference in the viewing of radiographs. There may be countless numbers present, making diagnosis simple, or the search may have to be a minute one for the identification of a single cysticercus in a very early stage of calcification. When this is found, and corresponds to any of the very definite shapes indicated, the diagnosis can be made. Solitary doubtful calcifications can be localized, excised, and, after solution of the calcium salts with weak hydrochloric acid, a search can be made for the hooklets of the scolex. Radiological examination at six-monthly intervals may be indicated in certain cases.

The advantages of diagnosing the presence of the condition are:

1. For the diagnosis of the actual com plaint.

2. For assessing the responsibility of service or employment for the presence of the disease -for example, epilepsy.

3. For assistance in the diagnosis of generalized or localized brain lesions.

4. To prevent operative treatment where the end-result is unlikely to be successful -for example, very heavy infestation.

5. To remove the hereditary stigma of " epilepsy."

I wish to acknowledge the help of Colonel W. .P. MacArthur, who is responsible for this investigation, the cooperation of Major H. B. F. Dixon, and the assistance of Captain F. P. M. Anderson in obtaining the clinical notes of the particular case described.

\section{ARTIFICIAL PNEUMOTHORAX IN THREE CASES OF PULMONARY TUBERCULOSIS IN CHILDREN}

BY

WILLIAM STOBIE, M.D.ED., M.R.C.P.

HONORARY PHYSICIAN, OSLER PAVILION FOR TUBERCULOSIS, RADCLIFFE INFIRMARY, OXFOKD

Pulmonary tuberculosis of the adult type with a positive sputum in children is generally recognized to be uncommon ; indeed, before the age of 10 it has been classed as " a medical curiosity." " While in the strictest sense this is hardly correct, the disease is unquestionably rare in the first decade of life. The outlook for these patients is grave. One authority ${ }^{2}$ on diseases of children says he has " never seen a case recover in which a definite diagnosis of pulmonary tuberculosis could be made." This experience is particularly unfortunate and not universal. A certain number of the afflicter? children do recover, and remain well.

In an endeavour favourably to influence the prognosis, collapse therapy was instituted in the following three patients, who were sent to the Osler Pavilion, Radcliffe Infirmary, by Dr. G. C. Williams, medical officer of health for the city of Oxford, to whom I am indebted for their early history. 


\section{CASE I}

A schoolgirl, aged 9, was admitted on January 28th, 1933. She was a sturdy child until she contracted whooping-cough in June, 1932. The cough persisted for a long time, and the child became listless and irritable. Her father had died from pulmonary tuberculosis three weeks before L.er admission to the Osler Pavilion; he had not sought medical advice till the day before death.

In addition to the cough there had been loss of weight, night sweats, and a small amount of morning sputum, in which tubercle bacilli were found. They were present in the sputum for several weeks. On examination an area of high-pitched bronchial breathing with consonating rales, and increasad tactile and vocal fremitus, were detected in the region of the angle of the right scapula, with a Skodaic note in the upper zone. There was also some crepitation in the right mid-zone anteriorly. The left lung was apparently unaffected.

Artificial pneumothorax was induced on February 3rd, 1933, and is being maintained with refills of 400 or $500 \mathrm{c} . \mathrm{cm}$. of air at fortnightly intervals. The child has been kept in hospital much longer than is customary, because of unfavourable home conditions. She is very well, has no cough or sputum, and has gained a great deal of weight.

\section{CASE II}

A schoolboy, aged 12, was admitted on April 26th, 1932. There was a history of cough of four months' duration, some sputum, loss of weight, lassitude, and dyspncea. Tubercle bacilli were found in the sputum.

The patient's mother had tubercle bacilli in the sputum. One sister, aged 17, had been in the Osler Pavilion the previous year with a positive sputum, and had been treated by artificial pneumothorax. In her case the collapse was not complete in the upper zone, and effusion subsequently developed in the pleural cavity. This cleared up in time and the patient is now at work, although the general condition is not altogether satisfactory on account of the contralateral disease (which was present on admission to hospital) being still active.

On examination of the boy signs of infiltration and breaking down were found in the right upper zone and in the right mid-zone in the axilla. The left upper zone was also slightly involved, as evidenced by a fair number of crepitations in that area.

Treatment by artificial pneumothorax was begun on May 2nd, 1932, and is being maintained in the out-patient department at intervals of about four weeks, with refills of 700 to 800 c.cm. of air. Apart from a fine adhesion at the apex it is satisfactory. During his stay in hospital the patient was given a course of sanocrysin, with the object of clearing up the disease in the left lung. At present there is no cough or sputum. The patient has gained nearly a stone in weight since his discharge, and the left side for the moment shows no signs of activity, although it must remain a source of anxiety.

\section{CASE III}

A girl, aged 15, was admitted on October 12th, 1932. Her illness began with a persistent cough following an attack of whooping-cough in July, 1932. Early in October the sputum, which had been present since the whooping-cough, was examined and found to contain tubercle bacilli. This finding was verified soon after the patient's admission to hospital. Examination of the chest revealed impairment of percussion note, weak "blowing" breath sounds, with crepitations in the left upper zone. There were one or two crepitations in the right apex.

An artificial pneumothorax was induced on October 28th, 1932, and the usual procedure of refilling it lengthening intervals was adopted. Complete collapse of the lung was prevented by fine adhesions from the apex to the chest wall. Since the discharge of the patient 700 to $800 \mathrm{c.cm}$. of air have been introduced into the left pleural cavity at intervals of approximately three weeks. A small " puddle" made its appearance oil August 23rd, 1933, and is still present. The right lung has remained "satisfactory." There has been no appreciable increase in weight for the past few weeks, presumably because of the small pleural effusion, but since discharge from the hospital there has been a gain of well over a stone.

It is much too early to talk about permanent results. All that can be claimed so far is that, in all probability, by having had given to them the advantage of modern methods of treatment, these young patients have escaped, for the time at least, the usual fate of sputum-positive cases at their respective ages.

\section{ReFerences}

Bentley, F. J.: Lancet, 1932, i, 689.

Findlay, L.: P'ost-Graduate Med. Journ., 1933, ix, 377.

\section{Memoranda MEDICAL, SURGICAL, OBSTETRICAL}

\section{$X$-RAY SICKNESS TREATED SUCCESSFULLY WITH LIVER EXTRACT}

In 1924 E. C. Dodds and I published a synopsis of a detailed clinical and biochemical study of $x$-ray sickness. We were able to show that a theory that it was due to an "acute uraemia" received no support whatever from metabolic studies, but we are not able to elucidate the essential cause. Sedatives, salines, alkalis, and glucose have all had apparent occas:onal success in treatment, but, so far as I know, no one in the last ten years has advanced a theory of cause and cure which is both unassailable and generally' applicable, until (as I noted in a recent letter to the Journal) Dietel ${ }^{2}$ reported his success with all of fifteen patients, following intramuscular injections of liver extract. He considers the sickness and recovery to be due to changes in the blood cholesterol, but unfortunately gives no figures as to the variations found. All his patients were gynaecological, and had abdominal radiation. Injections of 2 to 4 c.cm. were given.

Since November 2nd I have treated ten patients who complained of nausea, sickness, or prostration to more than a trivial degree. They were about a tenth of those treated in the six weeks with medium or deep $x$ rays: four were having medium-voltage post-operative treatment after radical mastectomy six were having high-voltage $x$ rays, foraortic gland metastasis (ovarian or testicular) and for spinal or other metastasis or primary breast cancer. In all but two the response has been both immediate and satisfactory: all the unpleasant effects vanished, and the patients continued the course of treatment in their usual health and spirits. One exception has been an out-patient who has suffered all her life from " livery attacks" ; she is also obliged to take an hcur's train journey immediately after the treatments. Injections of $2 \mathrm{c.cm}$. have not been enough for her certainly (Dietel has repeated $2 \mathrm{c.cm}$. injections sometimes, and latterly has given $4 \mathrm{c.cm}$. as a routine in all cases). The other exception was psychologically sick, sometimes even before a treatment caurse began.

To confirm or modify the theory of Dietel a research has been initiated with colleagues as to the blood changes resulting from the radiation and the liver injections. While awaiting the results of these invest.gations, it is of interest to note that clinical tests confirm his observations

${ }^{1}$ Dodds and Webster: Lancei, 1924, i, 533

2 Dietel: Strahlenth., September, 1933, Bd. 48, S.110. 